\title{
The Relationship between Dental Anxiety and Reported Dental Treatment Experience in 11-14-Year-Old Jordanian Children
}

\author{
Rania Abdalla Al-Saddi ${ }^{1}$ and Maan Yacoub Alfar ${ }^{2}$ \\ Department of conservative and pediatric dentistry, King Hussein Medical Center, Queen Rania Al Abdallah Hospital, Royal \\ Medical Service, Jordan Armed Forces.
}

\begin{abstract}
Background: Anxiety is a major drawback for children encountering different dental experiences and procedures. Aim: To determine the influence of reported dental experiences on dental anxiety in Jordanian children aged 11-14 years old. Design: From 630 children, aged 11-14 years, 583 (92.5\%) completed a two-part questionnaire. The first part was to record which dental procedures they had experienced. These procedures were the same as those included in the MCDAS (modified child dental anxiety scale), which formed the second part of the questionnaire. The scale consisted of eight questions, which invited the children to rate their anxiety about a variety of dental experiences ranging from attendance to the dentist to dental treatment under inhalation sedation. Results: Females were found to have a higher MDAS (mean dental anxiety score) than males $(p<0.05)$. Children were significantly less anxious about specific items of dental treatment if they had experienced that specific type of treatment. The MDAS for children who experienced restorative treatment was lower than those who reported no experience. Conclusions: Girls were more dentally anxious than boys and children who experienced dental visits more frequently were significantly less anxious than those who reported infrequent visits.
\end{abstract}

Key words: Dental Anxiety, Relationship, Dental treatment, Experience.

\section{Introduction}

Dental anxiety is a detrimental factor in dental treatment; it prevents many potential patients from seeking dental care and causes stress to the dentist delivering dental treatment. A major source of stress for dental practitioners is extremely uncooperative patient. Therefore the management of young children can be challenging for the dental practitioner as the level of anxiety increases due to their limited cooperation.

The concepts of fear and anxiety are closely associated and have different definitions. Geer [1] stated that the difference between fear and anxiety is thus conceptualized as a difference in the specificity of the stimulus. Fear is a response to a specific stimulus

Corresponding author: Rania abdalla Al-Saddi. BDs.Jordanian Dental Board in Conservative Dentistry, senior specia;ist,. Maan Yacoub Alfar, BDs,MDENTSie in Pediatric Dentistry (Liverpool, UK) Jordanian Dental Board, Consultant. while anxiety is a response to a more general or pervasive stimulus. Corah et al. [2] stated that dental anxiety is more specific than general anxiety; it is the patient's response to the stress specific to the dental situation.

Early surveys indicated that $5 \%-6 \%$ of the adult population avoided dental treatment because of extreme fear; the range may be as high as $16 \%$ among school-age children [3, 4]. Bedi et al. [5] investigated the prevalence of dental anxiety among 13-14 years old children and found that the prevalence of high dental anxiety was $7.1 \%$ with a higher level among girls and among children with lower social classes. Furthermore, dental anxiety which is a fairly common condition in 5 -year-old children is also closely associated with symptoms, irregular attendance pattern, history of extraction and having dentally anxious parents [6].

Gender in relation to the causes of dental anxiety has been thoroughly investigated. Ollendick et al. [7] 
found that females reported higher level of fear than males. Interestingly, boys endorsed more direct conditioning and vicarious conditioning sources than girls. Boys were more likely to report fear than girls when they had direct or vicarious conditioning experiences with the feared stimulus. On the other hand, girls reported fears that were largely due to instructional/informational sources. Effects due to nationality were minimal [7].

Questionnaires and rating scales are commonly used to measure dental anxiety in children [8]. Questionnaires can only be used with teenagers and adults as of the limitation of vocabulary, understanding and emotional development make these questionnaires inapplicable in younger children [9]. The evaluation of anxiety in children is based on observed behaviour using rating scales (behavioural ratings); Frankl scale is one of the rating and objective scales, which comprises of four ratings ranging from definitely negative to definitely positive [10].

The aim of the study was to determine the influence of reported dental experiences on dental anxiety in children aged 11-14 years old in Jordanian children.

\section{Materials and Methods}

Children aged 11-14 years (seventh, eighth and ninth school grades) attending public middle schools in the capital Amman coming from different social backgrounds were involved in this study.

After obtaining the ethical committee approval of Jordanian Royal Medical Services to conduct the study, a letter with consent form was distributed through the school to all parents of children participating in this study. The letter outlined the purpose and nature of the study and gave the opportunity to the parents not to participate if they had any objections. Only children with signed consent forms were allowed to fill the questionnaires.

A two-part questionnaire was used for the purpose of this study. In first part, an MCDAS (modified child dental anxiety scale) was employed to assess the child's dental anxiety. The scale consisted of eight questions, which asked the children to record which dental procedures they had experienced and included one or combination of the following experiences:

(1) General attendance to the dentist

(2) Dental examination

(3) Scaling and polishing

(4) Local anaesthesia

(5) Dental restoration

(6) Dental extraction

(7) Dental treatment under inhalation sedation

(8) Dental treatment under general anaesthesia

While the second part of the questionnaire allowed the children to report on a five-point Likert scale about how relaxed or worried they were for each of these scenarios. Children were asked to choose an answer which best sums up their feelings. The answers were scored from 1 to 5 and they would mean:

(1) Relaxed and not worried at all

(2) Slightly worried

(3) Moderately worried

(4) Fairly worried

(5) Extremely worried

Teachers distributed the questionnaire to children in their classes and each class teacher explained the purpose of the questionnaire. Children were invited to complete the questionnaire in their classes in the presence of the teacher. All the classes took place at the same time to prevent children discussing the questionnaire among each other. The teacher took the children through the questionnaire by reading all the questions and showing the children how they could make their answers, so that any misunderstandings in the comprehension of the questionnaire were solved at the time of completion by the help of teacher or head teacher.

\subsection{Statistical Analysis}

Data from questionnaires were compiled using SPSS statistical software package for windows (version 17.0, SPSS Inc., Chicago, IL, USA). Comparison of different 
variables was analyzed using $t$-test to determine the relationship between dental anxiety and reported dental experiences. Significance level was set at $p$-value of < 0.05 using two-tailed $t$-test throughout.

\section{Results}

Informed consent was gained from all participants ( $n$ $=630)$ of whom $583(92.5 \%)$ completed the questionnaire and $47(7.5 \%)$ did not participate due to absence. The age distribution of the participants is presented in Table 1.

Girls have shown higher MDAS (mean dental anxiety score) compared to boys (Table 2 ) reaching to a statistically significant correlation with dental anxiety ( $p<0.0001$, two-tailed $t$-test).

Table 3 demonstrates the relationship between dental anxiety and exposure to different dental experiences among the participants. In all types of dental experiences and procedures children who experienced a specific type of dental experience or procedure had lower MDAS compared to children who did not undergo a similar dental experience or procedure. The lowest MDAS was for children who underwent dental examination followed by attendance to dentist experience. Furthermore, MDAS was highest for children who did not experience local anaesthesia

Table 1 Age group distribution of the participants.

\begin{tabular}{llll}
\hline Variable & Category & $N$ & $\%$ \\
\hline Age (years) & 11 & 130 & 22.5 \\
& 12 & 134 & 22.9 \\
& 13 & 145 & 24.8 \\
& 14 & 174 & 29.8 \\
Total & & 583 & 100 \\
\hline
\end{tabular}

Table 2 Gender distribution and relationship with dental anxiety of the participants.

\begin{tabular}{lllll}
\hline Variable & Category & $n$ & $\%$ & MDAS \\
Gender & Boys & 345 & 59.1 & $16.24 \pm 4.86^{*}$ \\
& Girls & 238 & 40.9 & $19.18 \pm 4.59^{*}$ \\
Total & & 583 & 100 & \\
\hline
\end{tabular}

* Significance level $p<0.0001, t$-test (two-tailed).

Table 3 Relationship of dental anxiety and exposure to different types of dental experiences.

\begin{tabular}{|c|c|c|c|c|}
\hline Type of experience & & $n(\%)$ & $\begin{array}{l}\text { MDAS } \\
\text { Mean } \pm \text { SD }\end{array}$ & $p$-value* \\
\hline \multirow[t]{2}{*}{ Attendance to dentist } & Yes & $329(58.3)$ & $2.17 \pm 7.13$ & \multirow{2}{*}{0.396} \\
\hline & No & $236(41.7)$ & $2.28 \pm 8.02$ & \\
\hline \multirow[t]{2}{*}{ Dental examination } & Yes & $429(82.1)$ & $2.08 \pm 1.06$ & \multirow{2}{*}{0.050} \\
\hline & No & $94(17.9)$ & $2.30 \pm 0.99$ & \\
\hline \multirow[t]{2}{*}{ Scaling and polishing } & Yes & $234(48.5)$ & $2.24 \pm 1.14$ & \multirow{2}{*}{0.662} \\
\hline & No & $248(51.5)$ & $2.29 \pm 1.22$ & \\
\hline \multirow[t]{2}{*}{ Local anaesthesia } & Yes & $376(66.1)$ & $3.59 \pm 1.17$ & \multirow{2}{*}{0.947} \\
\hline & No & $193(33.9)$ & $3.68 \pm 1.25$ & \\
\hline \multirow[t]{2}{*}{ Dental restoration } & Yes & $318(56.9)$ & $2.42 \pm 1.30$ & \multirow{2}{*}{0.004} \\
\hline & No & $240(43.1)$ & $2.74 \pm 1.20$ & \\
\hline \multirow[t]{2}{*}{ Dental extraction } & Yes & $319(56.1)$ & $3.59 \pm 1.34$ & \multirow{2}{*}{0.832} \\
\hline & No & $250(43.9)$ & $3.62 \pm 1.28$ & \\
\hline \multirow[t]{2}{*}{$\begin{array}{l}\text { Dental treatment under inhalational } \\
\text { or General Anaesthesia }\end{array}$} & Yes & $112(21.9)$ & $2.63 \pm 1.48$ & \multirow{2}{*}{0.000} \\
\hline & No & $398(78.1)$ & $3.30 \pm 1.50$ & \\
\hline
\end{tabular}

* Significance level $p<0.05, t$-test (two-tailed). 
followed by those who did not experience dental extraction. Although children who reported attendance within six months had lower MDAS, no statistical significant correlation was found between those who had regular attendance and those who had irregular attendance to dentist. No statistical significant correlation was also found in children who underwent scaling and polishing, local anaesthesia, and dental extraction with children who did not undergo a similar dental procedure. On the other hand, significant statistical correlations were evident in children who underwent dental examination, dental restoration, and dental treatment under general anaesthesia when compared to children who did not undergo a similar dental experience or procedure.

\section{Discussion}

The aim of this study was to determine the influence of reported dental experience on dental anxiety in children aged 11-14 years old in Amman the capital city of Jordan.

Dental anxiety was reported relating to: attending to dentist in general, dental examination (check-up), scaling and polishing, local anaesthesia, dental filling, dental extraction and dental treatment under general anaesthesia. Dental treatment under inhalation sedation was excluded from the scale in order not to be mixed up with general anaesthesia by children. The MCDAS that we used in this study has been reviewed for reliability and proved its validity to be used for measuring dental anxiety $[11,12]$. The multivariate analysis showed that the MDAS for boys was less than the value for girls. This reached statistical significance, which indicates that dental anxiety was higher in girls than boys within this study group. The same gender difference has been demonstrated in a number of studies [5, 13]. One study had also shown that dental anxiety both before and after treatment was higher among girls than among boys [14]. Carrillo-Diaz et al. [15] in their study had stated that dental fear and/or anxiety associated with dental visit is connected with gender and appears to have negative impact on girls, who reported higher level anxiety and negative emotional outcomes compared with boys. Further study by Liddell [16] suggested that these differences may indicate a tendency for girls to be more influenced by internal factors, whereas boys react to a greater extent to external stresses than girls. Regular dental attendance to dentist and dental examination has been claimed by many authors to be a factor for decreasing dental anxiety due to the repeated exposure to dental examination and treatment and the experience gained by the children from increasing access to dental care [17]. Irregular attendees to dental examination and treatment usually present the dental office when there is an urgent oral health problem which needs invasive and traumatic procedure which reflects the level of anxiety to those groups of children. Studies have demonstrated that there is strong link between regular and irregular attendance in relation to dental anxiety [13, 16]. Wigen et al. [18] found that irregular attendees had a significantly higher (dmft), those in whom parents avoid bringing their children to scheduled dental appointments and child behaviour management problems in early dental visits increase the risk of having caries experience and dental anxiety. This may be interpreted as dental anxiety increasing after experience of traumatic dental procedures compared with receiving non-traumatic procedure [18]. McGrath et al. [19] found that those experiences of high level of dental anxiety are among those with irregular attendance and the poorest oral health-related to quality of life in Britain [19]. Leroy et al. [20] in their study concluded that parents of young children need to be informed about motivation for an early dental visit. Promotion campaigns should focus on first born children, children from less educated parents, and children who do not regularly attending a dentist [20]. It was found in this study that there was no statistically significant relationship between children's experience of scale and polish, and their specific anxiety about scale and polish. Children who had experienced scale 
and polish were less anxious than those who had not experienced. The reason can be explained by that children who receive some minimal invasive dental treatment have become more experienced and less anxious than those who have not received any treatment [21]. Chadwick [22] assessing the anxious patient also found that the patient who returns to the dentist suggests that they trust the dentist and are trying to find ways of dealing with their anxieties. In the relationship between children who had experienced local anaesthesia and those who had not, it was found that there was no statistical significant relationship between children's experience of local anaesthesia and their anxiety about local anaesthesia. Surprisingly in this study the children with previous experience of local anaesthesia were less anxious about this than those who had not experienced local anaesthesia. However, Humphris et al. [23] found that a strong association between a traumatic treatment intervention and dental anxiety. They pointed out that in their results local anaesthesia in particular $(p<0.001)$ had higher statistical significance [23]. A similar study found a strong association between dental anxiety and BIIP (blood, injury, injection phobia). The results indicate that among adolescents BIIP is relatively often connected with dental anxiety [24]. Through the result of this study supported by Moore et al. [25], they found that the majority of traumatic dental experiences could not be directly linked to pain, but rather the dentist's attitude or the atmosphere in the clinic. Carlsen et al. [26] also found in their study that children, who were asked before and after treatment about both pain and dental anxiety, reported significantly less dental anxiety than the control group. One explanation could be that the treatment approach adopted by the majority of the general dental practitioners and paediatric dentists was atraumatic or less traumatic by using for example topical anaesthetic and behaviour management approach (tell-show-do, acclimatisation, etc.). This study also compared children who had experienced restorative care with those who had not.
There was a statistically significant relationship between children's experience of restorative treatment, and their specific anxiety about restorations. It was found that the group of children with previous history of experiencing restorative treatment had a lower mean anxiety score for this item of the questionnaire than those who had not experienced a restorative treatment. This reached statistical significance. This result is consistent with other finding. Milsom et al. [6] stated that the restorative approach adopted by the majority of general dental practitioners is atraumatic for children and also that restorative procedures may well be less traumatic for children than the procedures required to undertake an extraction under either local anaesthesia or general anaesthesia. Schriks and van Amerongen [27] also concluded that children treated according to the ART (atraumatic restorative treatment) approach using hand instruments alone experience less anxiety and discomfort than those treated using rotary instruments [27]. When children who had experienced a dental extraction were compared with those who had not, there was no statistically significant relationship between children's experience of dental extraction and their anxiety about dental extraction. It was found that those children who had history of extraction had a lower mean anxiety value than children who had not experienced this form of treatment. It is generally believed that extraction is a traumatic procedure for young children. Milsom et al. [6] found that children who had a history of extraction were three and a half times more likely to be anxious than children who had no experience of this form of treatment. Other study concluded that invasive dental treatments were rated the most intense among dental treatment pains and girls were commonly inclined to report more frequent and more intense pain compared with boys [28]. However, this result in the study group of Jordanian children could be explained by the findings of other studies. Siddle and Remington [29] stated that children might not acquire dental anxiety if they had a traumatic, painful experience after some relatively painless dental 
treatment, prior to the painful experience [29]. Other complementary therapies such as relaxation and breathing techniques (meditation) also have been successfully used in the management of dental anxiety with patients who are fearful of receiving dental treatment [30]. When comparing children who had experienced dental treatment under general anaesthesia and those who had not, it was found that there was a statistically significant relationship between children's experience of dental treatment under general anaesthesia, and their anxiety. Children who had experienced treatment under general anaesthesia had a lower mean anxiety score for this item of the questionnaire than those who had not experienced treatment under general anaesthesia. This result could be explained by a model of latent inhibition, and it may be because older children have had the chance of more dental visits of positive nature and this has helped to reduce this anxiety. Therefore older children tend to lose their anxiety, while younger children have not yet this opportunity [31]. It can be postulated that dental anxiety may disappear, not as a result of losing the fear-conditioned reflex, but because the unconditioned reflex evoked by the phobic situation has been reassessed. However, Arch et al. [32] in the study of children choosing between general anaesthesia or inhalation sedation for dental extraction, found no increase in the mean value for dental anxiety following general anaesthesia when compared to anxiety before general anaesthesia [32].

\section{Conclusion}

Conclusions were based on mean values for dental anxiety after children had been grouped according to their gender or reported dental anxiety. Dental anxiety is a fairly uncommon condition in 11-14 year-old children attending public schools in Amman the capital city of Jordan. Girls were found to be statistically significantly more dentally anxious than boys $(p<$ $0.05)$. Children who reported that they had visited the dentist more frequently were statistically significantly less anxious than those who reported infrequent visits. Children were statistically significantly less anxious about specific items of dental treatment if they had experienced that particular form of treatment $(p<0.05)$. This applied to: Dental examination, Restorative treatment and Dental treatment under general anaesthesia.

\section{Recommendation}

The pattern of sporadic attendance and care can setup a vicious cycle leading to long lasting dental anxiety problems. In order to prevent anxiety, we must break this cycle by making the dental experience easier and more pleasant for children and by providing effective public health measures to prevent dental disease.

\section{Why This Article Is Important}

- This article presents a thorough analysis of 7 aspects of dental treatment that cause dental anxiety in Jordanian children, which is the first article of its kind in Jordan.

- This article emphasizes the importance of regular visits to dental clinic as an approach for reducing dental anxiety, particularly in girls where they showed increased level of anxiety compared to boys.

- This article sets foundation for behavioural management policies in Jordanian children who are exposed to previous traumatic dental experience and increased level of dental anxiety.

\section{References}

[1] Geer, J. H. 1965. "The Development of a Scale to Measure Fear." Behav Res Ther 3: 45-53.

[2] Corah, N. L., Gale, E. N., and Illig, S. J. 1978. “Assessment of a Dental Anxiety Scale." J Am Dent Assoc 97: 816-9.

[3] Kent, G. G., and Blinkhorn, A. S. 1991. The Psychology of Dental Care, 2nd ed. Bristol: Wright, 55-76.

[4] Stricker, G., and Howitt, J. W. 1965. "Physiological Recording during Simulated Dental Appointments." NY State Dent J 31: 204-6.

[5] Bedi, R., Sutcliffe, P., Donnan, P. T., and McConnachie, J. 1992. "The Prevalence of Dental Anxiety in a Group of 13- and 14-Year-Old Scottish Children." Int J Paediatr 


\section{Experience in 11-14-Year-Old Jordanian Children}

Dent 2: 17-24.

[6] Milsom, K. M., Tickle, M., Humphris, G. M., and Blinkhorn, A. S. 2003. "The Relationship between Anxiety and Dental Treatment Experience in 5-Year-Old Children." Br Dent J 194: 503-6.

[7] Ollendick, T. H., Yule, W., and Ollier, K. 1991. "Fears in British Children and Their Relationship to Manifest Anxiety and Depression." J Child Psychol Psychiatry 32: 321-31.

[8] Corah, N. L. 1969. "Development of a Dental Anxiety Scale.” J Dent Res 48: 596.

[9] Cuthbert, M. I., and Melamed, B. G. 1982. "A Screening Device: Children at Risk for Dental Fears and Management Problems." ASDC J Dent Child 49: 432-6.

[10] Frankl, S. N., Shiere, F. R., and Fogels, H. R. 1962. "Should the Parent Remain with the Child in the Dental Operatory?" J Dent Child 2: 150-63.

[11] Humphris, G. M., Morrison, T., and Lindsay, S. J. 1995. "The Modified Dental Anxiety Scale: Validation and United Kingdom Norms." Community Dent Health 12: 143-50.

[12] Humphris, G. M., Freeman, R., Campbell, J., Tuutti, H., and D'Souza, V. 2000. "Further Evidence for the Reliability and Validity of the Modified Dental Anxiety Scale." Int Dent J 50: 367-70.

[13] Quteish Taani, D. S. 2002. "Dental Anxiety and Regularity of Dental Attendance in Younger Adults." $J$ Oral Rehabil 29: 604-8.

[14] Toledano, M., Osorio, R., Aguilera, F. S., and Pegalajar, J. 1995. "Children's Dental Anxiety: Influence of Personality and Intelligence Factors." Int J Paediatr Dent 5: 23-8.

[15] Carrillo-Diaz, M., Crego, A., and Romero-Maroto, M. 2013. "The Influence of Gender on the Relationship between Dental Anxiety and Oral Health-Related Emotional Wellbeing." Int J Paediatr Dent 23: 180-7.

[16] Liddell, A. 1990. "Personality Characteristics versus Medical and Dental Experiences of Dentally Anxious Children." J Behav Med 13: 183-94.

[17] Sohn, W., and Ismail, A. I. 2005. "Regular Dental Visits and Dental Anxiety in an Adult Dentate Population." J Am Dent Assoc 136: 58-66.

[18] Wigen, T. I., Skaret, E., and Wang, N. J. 2009. "Dental Avoidance Behaviour in Parent and Child as Risk Indicators for Caries in 5-Year-Old Children." Int $J$ Paediatr Dent 19: 431-7.

[19] McGrath, C., and Bedi, R. 2004. "The Association between Dental Anxiety and Oral Health-Related Quality of life in Britain." Community Dent Oral Epidemiol 32: 67-72.

[20] Leroy, R., Bogaerts, K., Hoppenbrouwers, K., Luc, C., and
Dominique, D. M. 2013. "Dental Attendance in Preschool Children-A Prospective Study." Int J Paediatr Dent 23: 84-93.

[21] Murray, P., Liddell, A., and Donohue, J. 1989. “A Longitudinal Study of the Contribution of Dental Experience to Dental Anxiety in Children between 9 and 12 Years of Age." J Behav Med 12: 309-20.

[22] Chadwick, B. L. 2002. "Assessing the Anxious Patient." Dent Update 29: 448-54.

[23] Humphris, G. M., Mair, L., Lee, G. T. R., and Birch, H. 1991. "Dental Anxiety, Pain and Uncooperative Behaviour in Child Dental Patients." Anx Res 4: 61-77.

[24] Vika, M., Skaret, E., Raadal, M., Ost, L. G., and Kvale, G. 2008. "Fear of Blood, Injury, and Injections, and Its Relationship to Dental Anxiety and Probability of Avoiding Dental Treatment among 18-Year-Olds in Norway." Int J Paediatr Dent 18: 163-9.

[25] Moore, R., Brødsgaard, I., and Birn, H. 1991. "Manifestations, Acquisition and Diagnostic Categories of Dental Fear in a Self-referred Population." Behav Res Ther 29: 51-60.

[26] Carlsen, A., Humphris, G. M., and Lee, G. T. R. 1993. "The Effect of Pre-treatment Enquiries on Child Dental Patient's Post-treatment Ratings of Pain and Anxiety." Psychology and Health 8: 165-74.

[27] Schriks. M. C., and van Amerongen, W. E. 2003. "Atraumatic Perspectives of ART: Psychological and Physiological Aspects of Treatment with and without Rotary Instruments." Community Dent Oral Epidemiol 31: $15-20$.

[28] Krekmanova, L., Bergius, M., Robertson, A., et al. 2009. "Everyday and Dental-Pain Experiences in Healthy Swedish 8-19 Year Olds: An Epidemiological Study." Int J Paediatr Dent 19: 438-47.

[29] Siddle, D. A. T., and Remington, B. 1987. "Latent inhibition and human pavlovian conditioning: research and relevance." In Cognitive Processes and Pavlovian Conditioning in Humans, edited by G. C. L. Davey. Chichester: John Wiley \& Sons Inc, 115-46.

[30] Hainsworth, J. M., Moss, H., and Fairbrother, K. J. 2005. "Relaxation and Complementary Therapies: An Alternative Approach to Managing Dental Anxiety in Clinical Practice." Dent Update 32: 90-2, 94-6.

[31] Davey, G. C. 1989. "Dental Phobias and Anxieties: Evidence for Conditioning Processes in the Acquisition and Modulation of a Learned Fear." Behav Res Ther 27: 51-8.

[32] Arch, L. M., Humphris, G. M., and Lee, G. T. 2001. "Children Choosing between General Anaesthesia or Inhalation Sedation for Dental Extractions: The Effect on Dental Anxiety." Int J Paediatr Dent 11: 41-8. 